Mannheimer Manuskripte zu Risikotheorie,

Portfolio Management und Versicherungswirtschaft

\title{
Nr. 123 \\ Zur Quantifizierung der Risikoprämien deutscher Versicherungsaktien im Kontext eines Multifaktorenmodells
}

\author{
Von \\ Rolf ElgEtI UND RAIMOND MAURER
}

Mannheim 09/2000 


\title{
Zur Quantifizierung der Risikoprämien deutscher Versicherungsaktien im Kontext eines Multifaktorenmodells
}

\author{
Von \\ Rolf Elgeti (London) und Raimond Maurer (Frankfurt) ${ }^{*}$
}

Zusammenfassung

Vorgestellt wird eine empirische Studie, welche den Zusammenhang zwischen Rendite und Risiko für ein Sample deutscher Versicherungsaktien im Zeitraum 1975-1998 untersucht. Als Methode wurde ein Multifaktorenmodell mit makroökonomischen Faktoren verwendet. Je nach Untersuchungszeitraum beläuft sich der Anteil der erklärten Varianz auf 9,29\% bis 13,62\%. Es konnte eine signifikanter negativer Einfluß zwischen der Veränderung des allgemeinen Zinsniveaus und den Risikoprämien von Versicherungsaktien identifiziert werden. Weiterhin ist Wechselkurses der DM zum US-Dollar signifikant

\begin{abstract}
In this contribution we present an empirical study that focuses the relationship between risk and return for a universe of insurance stocks in Germany during the period 1975-1998. The study is motivated by the use of a multi factor model. The proportion of explained variance ranges from $9,29 \%$ to $13,62 \%$ in the monthly regressions. We find a significant and negative relationship between changes in the term structure of interest rates and the risk premiums for insurance stocks. Additionally, the exchange rate of the Deutsche Mark against the US-Dollar.
\end{abstract}

Schlagwörter

Risikoprämien, Versicherungsaktien, Kapitalmarkt.

Keywords

Risk Premiums, Insurance Stocks, Capital Markets

*) Die Arbeit wurde dankenswerterweise durch die Deutsche Forschungsgemeinschaft SFB 504 unterstützt. Einem anonymen Gutachter, Peter Albrecht und Frank Reiner danken wir für konstruktive Kritik. 


\section{Motivation}

Im Zentrum dieser Arbeit steht die Identifikation von ökonomischen Einflußfaktoren auf die Risikoprämien, also den Zusatzrenditen über den risikolosen Zinssatz hinaus, von deutschen börsengehandelten Versicherungsaktien. Die Differenzierung des Risikos von Versicherungsaktien in verschiedene ursächliche Faktoren ist aus mehreren Gründen von betriebswirtschaftlicher Bedeutung.

Zum einen ist im Kontext der aktionärsorientierten Steuerung von Versicherungsunternehmen die Quantifizierung der Eigenkapitalkosten, d.h. der von den Aktionären geforderten Rendite ihres Investments bezogen auf das eingegangene Risiko von zentraler Bedeutung. Dabei versuchen finanzierungstheoretische Ansätze die Ermittlung von risikoadjustierten Eigenkapitalkosten regelmäßig durch Rückgriff auf Kapitalmarktdaten zu objektivieren. ${ }^{1)}$ Auch bei der Übertragung finanzierungstheoretischer Überlegungen auf Fragen der Prämienkalkulation, ist die Bestimmung einer an den Verhältnissen des Kapitalmarkts orientierten riskoadjustierten Verzinsung des Aktionärskapitals relevant. Hierbei wird besonders in der US-amerikanischen Literatur im Rahmen sogenannter Financial Insurance Pricing-Modelle ein expliziter Kapitalmarktbezug bei der Prämienkalkulation gefordert. ${ }^{2)}$ In einigen Bundesstaaten der USA wurden solche Überlegungen überdies zur behördlichen Regulierung von Versicherungsprämien genutzt. ${ }^{3)}$

Im Zusammenhang mit M\&A-Aktivitäten in der Versicherungsbranche sowie beim Management von Beteiligungen an Versicherungsunternehmen ist der Einsatz von marktorientierten Unternehmensbewertungsmodellen bedeutungsvoll. Dabei wird nach der sogenannten Risikozuschlagsmethode die Unsicherheit zukünftiger finanzieller Überschüsse durch einen an das Risiko angepassten Diskontierungszinssatz berücksichtigt. ${ }^{4)}$ Dieser ermittelt sich als (landesüblicher)

1) Vgl. Schmalenbach-Gesellschaft 1996, S. 547.

2) Vgl. etwa Cummins/Harrington 1987, Cummins 1990 oder Derring 1990. In der deutschsprachigen (risikotheoretischen) Literatur wird etwa bei Mack 1997, S. 27 ein Bezug zum Kapitalmarkt bei der Prämienkalkulation eingeräumt. Eine kritische Auseinandersetzung hinsichtlich einer unreflektierten Übertragung von Erkenntnissen der Kapitalmarkttheorie auf versicherungswissenschaftliche Fragestellungen, insbesondere unter Nichtbeachtung risikotheoretischer Aspekte, findet sich in Albrecht 1991.

3) Vgl. Hill/Modigliani 1987 für Massachusetts.

4) Eine Übersicht verschiedener Discounted Cash Flow-Verfahren zur Unternehmensbewertung findet sich etwa in Hachmeister 1998, Ballwieser 1998, Böcking/Nowak 1998 sowie IDW ES1 1999. Eine Diskussion zwischen der Sicherheitsäquivalenz- und Risikozuschlagsmethode bei der Unternehmensbewertung findet sich in Schwetzler 2000. 
risikoloser Basiszins zuzüglich einer Risikoprämie, in der die Risikoaversion von Wirtschaftssubjekten erfaßt wird. Insbesondere für börsennotierte Unternehmen ist es zweckmäßig, Risikoprämien objektiviert durch Rückgriff auf Kapitalmarktdaten und Kapitalmarktmodelle abzuleiten.

Schließlich sind Informationen über den Zusammenhang zwischen Rendite und Risiko von Versicherungsaktien auch für das (institutionelle) InvestmentManagement von Relevanz. Anwendungen finden sich etwa bei der RenditeRisiko-Optimierung, der Performanceanalyse und -attribution oder der Bestimmung von Positionslimits entsprechender Branchenportfolios aus Versicherungsaktien. ${ }^{5)}$

Die moderne Finanzierungstheorie besagt nun, daß die erwarteten Renditen von börsengehandelten Aktien eine Funktion des vom Kapitalmarkt entlohnten Risikos darstellen. Dabei kennt das traditionelle Marktmodell als einzigen Risikofaktor die Abhängigkeit der Rendite einer Aktie von derjenigen des Gesamtmarktes, gemessen durch den Betafaktor. Allerdings zeigen viele empirische Tests, daß das Marktmodell die Renditen für Aktienmärkte im allgemeinen ${ }^{6)}$ und Versicherungsaktien im besonderen nicht ausreichend erklärt. ${ }^{7)}$ Insbesondere deuten die Studien darauf hin, daß neben dem Marktindex noch weitere Faktoren existieren, welche die Schwankung von Aktienrenditen signifikant erklären. Die in dieser Arbeit betrachteten Multifaktorenmodelle bieten eine differenziertere Art einen Bezug zwischen Rendite und Risiko von Versicherungsaktien herzustellen. Sie stellen insofern eine Verallgemeinerung des Marktmodells dar, als mehrere gemeinsame Risikofaktoren betrachtet werden, die jeweils separat am Kapitalmarkt entlohnt werden.

Der vorliegende Beitrag ist wie folgt disponiert: In Abschnitt zwei werden die theoretischen Grundlagen und die Möglichkeiten zur Identifikation von Multifaktorenmodellen erörtert. Im dritten Abschnitt wird die Datenbasis sowie das Untersuchungsdesign der empirischen Auswertungen vorgestellt. Der vierte Abschnitt enthält die Ergebnisse der empirischen Untersuchungen. Der abschließende fünfte Abschnitt faßt die Ergebnisse der Arbeit zusammen.

5) Vgl. hierzu etwa Albrecht/Maurer/Mayser 1996, S. 15ff. oder Mayser 1996, S. 36ff.

6) Vgl. für US-amerikanische Aktienmärkte etwa die Studien von Friend/Westerfield/Granito 1978, Fama/French 1992, 1993 und für den deutschen Aktienmarkt Möller 1985, S. 97ff. oder Frantzmann 1990. Zu einer theoretischen Kritik hinsichtlich der Beobachtbarkeit des Marktportfolios, vgl. Roll 1977.

7) Vgl. die Studien von Harrington 1983 bzw. Cummins/Harrington 1988 für Aktienrenditen von US-amerikanischen Lebens- bzw. Schadenversicherungsunternehmen. 


\section{Grundlagen von Multifaktorenmodellen}

\subsection{Formale Grundstruktur und Verbindung zur APT}

Multifaktorenmodelle postulieren, daß die zufallsabhängigen Einperiodenrenditen $R_{i}$ der $i=1, \ldots ., n$ Aktien eines betrachteten Marktes in einem linearen Modell durch gemeinsame fundamentale Faktoren erklärt werden können. ${ }^{8)}$ Formal haben Multifaktorenmodelle die folgende Struktur:

$$
R_{i}=a_{i}+\sum_{j=1}^{m} b_{i j} F_{j}+\varepsilon_{i}
$$

Dabei bezeichnet die Zufallsgröße $F_{j}(j=1, . ., m)$ den $j$-ten gemeinsamen Faktor des untersuchten Wertpapiermarktes und $\varepsilon_{i}$ die nicht durch die gemeinsamen Faktoren erklärbare zufallsabhängige Renditekomponente der $i$-ten Aktie, die sogenannte spezifische Rendite. Die Koeffizienten $b_{i j}$ quantifizieren die Sensitivität der Rendite der $i$-ten Aktie bezüglich des $j$-ten gemeinsamen Faktors und werden auch als Faktorladungen bezeichnet. Als strukturelle Annahmen werden getroffen: $\operatorname{Cov}\left(\varepsilon_{h}, \varepsilon_{i}\right)=0$ für alle $\mathrm{h} \neq \mathrm{i}$ und $\operatorname{Cov}\left(F_{j}, \varepsilon_{i}\right)=0$ für alle $i, j$. Das bedeutet, daß sowohl die spezifischen Renditen und die Faktoren als auch die spezifischen Renditen der einzelnen Titel jeweils unkorreliert sind. Damit ist die Korrelation zwischen den Aktienrenditen ausschließlich durch die gemeinsame Abhängigkeit von den Faktoren bedingt. Eine weitere Annahme ist, daß der Erwartungswert der spezifischen Rendite $\varepsilon_{i}$ gleich Null ist. Das hat zur Konsequenz, daß die gesamte erwartete Rendite, die nicht durch die Faktoren bedingt ist, in der Komponente $a_{\mathrm{i}}$ enthalten ist.

Multifaktorenmodelle stehen in enger Verbindung mit der Arbitrage Pricing Theory $(\mathrm{APT})^{9)}$ und erfahren damit - ähnlich wie das Marktmodell durch das CAPM - eine kapitalmarkttheoretische Fundierung. Die APT geht von der Annahme aus, daß die Renditen $R_{i}$ der Finanztitel eines gegebenen Marktes einem linearen Faktorenmodell der Form (1) folgen. Darüber hinaus wird von standardisierten und orthonormalen Faktoren ausgegangen, ${ }^{10)}$ was in der APT-

8) Vgl. im folgenden auch Albrecht/Maurer/Mayser 1996, S. 5ff., Mayser 1996, S. 289 ff. oder Stephan/Maurer 2000.

9) Vgl. Ross 1976, S. 341 oder Huberman 1982, S. $183 \mathrm{f}$.

10) Damit lassen sich die Annahmen der APT formal wie folgt darstellen: $\mathrm{E}\left(\varepsilon_{i}\right)=\operatorname{Cov}\left(\varepsilon_{i}, \varepsilon_{j}\right)=$ $\mathrm{E}\left(F_{k}\right)=\operatorname{Cov}\left(F_{k}, \varepsilon_{j}\right)=0$ für alle $i, j \neq i, k, l \neq k$ sowie $\operatorname{Var}\left(F_{k}\right)=1$ für alle $k$. Ein Faktorenmodell des Typus (1) läßt sich gewöhnlich in ein äquivalentes Faktorenmodell mit standardisierten und orthonormalen Faktoren transformieren, vgl. hierzu Albrecht/Maurer/Mayser 1996 oder Mayser 1996, S. $291 \mathrm{f}$. 
Literatur auch als striktes Faktorenmodell bezeichnet wird. ${ }^{11)}$ Auf der Grundlage eines strikten Faktorenmodells und mit Hilfe von Argumenten der Arbitragefreiheit von Wertpapiermärkten läßt sich die folgende Gleichung exakt oder in asymptotischer Form (für eine sehr große Anzahl $n$ von Wertpapieren) für die erwartete Rendite des Titels $i(i=1, \ldots, n)$ herleiten: $\left.{ }^{12}\right)$

$$
E\left(R_{i}\right)=\lambda_{0}+\sum_{k=1}^{m} \lambda_{k} b_{i k}
$$

Dabei entspricht $\lambda_{0}$ der Rendite einer risikolosen Anlage, falls eine solche am Markt existiert. ${ }^{13)}$ Die Faktoren $\lambda_{k}$ sind für alle Wertpapiere gleich und können unter gewissen Bedingungen ${ }^{14)}$ als Risikoprämie interpretiert werden, die ein Investor für das Eingehen eines Exposure zum Risikofaktor $k$ erwarten kann. Damit werden im Rahmen der APT (wie auch beim CAPM) nur systematische Risiken, d.h. solche welche die Investoren nicht durch Diversifikation eliminieren können, durch den Markt bewertet. Analog zum sogenannten Beta-Faktor des CAPM, welcher inhaltlich die vom Kapitalmarkt entlohnte Sensitivität gegenüber einem Gesamtmarkt darstellt, können die Faktorsensitivitäten der APT als Kenngrößen für das systematische Risiko aufgefaßt werden. Dabei existieren im Rahmen der APT-Preisgleichung mehrere Risikomaße, womit im Vergleich zum CAPM die erwartete Rendite eines Wertpapiers in differenzierter Weise ihrem Risiko zugeordnet wird. Im Gegensatz zum CAPM bleibt bei der APT allerdings offen, welche Faktoren konkret zu verwenden sind.

\subsection{Identifikation von Multifaktorenmodellen}

Auf der statistischen Ebene existieren verschiedene Ansätze zur Identifikation von Multifaktorenmodellen. Dabei stehen sowohl Zeitreihen- wie auch Querschnittsdaten zur Verfügung. Insofern läßt sich differenzieren in Methoden der Zeitreihenanalyse, der Querschnittsanalyse sowie Kombinationen von beiden.

11) Darüber hinaus spielen auch sogenannte approximative Faktorenmodelle, welche eine Abschwächung der Annahme unkorrelierter Residuen beinhalten, eine Rolle, vgl. etwa Chamberlain 1983, Chamberlain/Rothschild 1983 oder Mayser 1996, S. 292.

12) Dabei ist darauf hinzuweisen, daß die APT ohne Verteilungsannahme hinsichtlich der Wertpapierrenditen und mit schwachen Annahmen bezüglich der Präferenzen der Anleger auskommt. Alternativ läßt sich diese Gleichung exakt unter der stärkeren Annahme eines Kapitalmarktgleichgewichts herleiten; vgl. hierzu auch Shanken 1982.

13) Falls eine solche nicht existiert, bleibt dieser Summand trotzdem Bestandteil des Modells und ist als Rendite zu verstehen, die ein Anleger erwarten kann, wenn er sein Portefeuille keinen Risikofaktoren aussetzt.

14) Vgl. etwa Ingersoll 1987, S. 168. 
Mit Hilfe der statistischen Faktorenanalyse werden auf der Grundlage von Zeitreihen- und Querschnittsdaten die Faktoren, deren Anzahl und die Faktorladungen simultan identifiziert. ${ }^{15)}$ Als Vorteil ist die Objektivität des Verfahrens zu nennen. Weder Faktoren noch Sensitivitäten werden vorgegeben, sondern aus historischen Daten geschätzt, so daß die Vergangenheit optimal erklärt wird. ${ }^{16)}$ Das wesentliche Problem besteht darin, daß die extrahierten Faktoren und Faktorladungen lediglich statistische Konstrukte darstellen, deren ökonomische Interpretation nur bedingt möglich ist. ${ }^{17)}$

Bei Querschnittsanalysen werden die Faktorladungen als bekannt vorausgesetzt und eine Regression über alle Wertpapiere $\mathrm{zu}$ einem bestimmten Zeitpunkt durchgeführt. Dabei wird angenommen, daß sich die Faktorladungen durch fundamentale Daten des Unternehmens erklären lassen. In den grundlegenden Beiträgen von Rosenberg (1974) und Rosenberg/Marathe (1976) wurden hierzu unternehmensspezifische Kennzahlen für den Erfolg, die Größe oder den Verschuldungsgrad des Unternehmens herangezogen. ${ }^{18)}$ Dieser Ansatz besitzt neben seiner mikroökonomischen Fundierung den Vorteil der guten Identifizierbarkeit von zeitlichen Änderungen in den Koeffizienten. ${ }^{19)}$ Zentrales Problem ist die Spezifikation von geeigneten fundamentalen Erklärungsgrößen des Unternehmens und die damit verbundenen erheblichen Anforderungen an Qualität und Umfang der Daten.

Im Kontext der in dieser Arbeit verwendeten Zeitreihenanalysen geht man von bekannten (und damit direkt beobachtbaren), ökonomisch plausiblen Faktoren aus und schätzt die Matrix der Faktorladungen für die einzelnen Wertpapiere im Rahmen linearer Regressionsansätze. ${ }^{20)}$ Eine solche Methodik bietet den Vorteil der guten Interpretierbarkeit der ausgewählten Faktoren, deren empirische Relevanz unmittelbar statistisch überprüft wird. Weiterhin ist die Verwendung makroökonomischer Faktoren zur Erklärung der Renditeentwicklungen eines

15) Dabei wird etwa auf das Maximum-Likelihood- oder das Principal-Component-Verfahren zurückgegriffen, vgl. hierzu etwa Rao 1996.

16) Vgl. Connor 1995, S. 42.

17) Die simultane Identifikation von Faktoren und Faktorladungen spielt allerdings in empirischen Studien zum Test der APT-Gleichung eine bedeutende Rolle. Hinsichtlich Überlegungen zur Testbarkeit der APT sei auf Roll/Ross 1980 oder Shanken 1982 verwiesen. Eine Anwendung von Faktoranalysen zur Schätzung von Eigenkapitalkosten USamerikanischer Versicherungsaktien findet sich in Lee/Cummins 1995, S. $251 \mathrm{ff}$.

18) Von verschiedenen Investment Consulting-Unternehmen werden fundamentale Multifaktorenmodelle als Beratungstools für die Steuerung von Aktienportfolios institutioneller Anleger eingesetzt, vgl. etwa BARRA 1991 oder Rudd/Clasing 1988.

19) $\mathrm{Zu}$ einer vergleichenden Studie der verschiedenen Ansätze vgl. Beckers et al. 1993 für den schweizerischen und Connor 1995 für den amerikanischen Aktienmarkt.

20) Vgl. Connor 1995, S. 42f. 
Aktienmarktes intuitiv und bietet den Vorteil der guten Verfügbarkeit von öffentlich zugänglichen Daten. Zentrales Problem ist die Annahme, daß die Faktorladungen über den Zeitablauf konstant bleiben. Vielmehr ist es plausibel, daß die Sensitivität einer Aktie gegenüber einem Faktor zeitlichen Änderungen unterliegt. Dem kann etwa durch Unterteilung des Untersuchungszeitraums in Teilperioden Rechnung getragen werden. ${ }^{21)}$

Typischerweise werden in empirischen Studien zur Identifikation von Multifaktorenmodellen auf der Basis von Zeitreihenanalysen makroökonomische Faktoren verwendet. So zeigen etwa Chen/Ross/Roll (1986) und Berry/Burmeister/McElroy (1988) auf, daß der Renditespread zwischen lang- und kurzfristigen Zinstiteln, die antizipierte bzw. nicht-antizipierte Entwicklung der Inflationsrate, die Veränderung der Industrieproduktion sowie die der RenditeSpread zwischen Anleihen unterschiedlicher Bonität einen signifikanten Einfluß auf die Renditen von US-amerikanischen Aktien aufweisen. ${ }^{22)}$ Speziell für Versicherungsaktien existiert eine Studie von Mei/Saunders (1994) für den amerikanischen Markt. Dabei verwenden die Autoren als Erklärungsvariablen eine Dummy-Variable für den Monat Januar, einen kurzfristigen Zins, den Zinsspread sowie eine allgemeine Dividenden- und eine Immobilienrendite. Die Untersuchungsergebnisse zeigen, daß mit Ausnahme der Januar-Dummies und des Zinsspreads alle Faktoren einen signifikanten Einfluß auf die Risikoprämien von Versicherungsaktien haben. Dies wird im weiteren für die vorliegende Arbeit von Bedeutung sein.

\section{Datenbasis und Untersuchungsdesign}

\subsection{Auswahl der Aktien}

Grundlage für die nachfolgenden empirischen Auswertungen sind monatliche Kurs- und Dividendendaten von deutschen Versicherungsaktien im Zeitraum 01/1975 bis 12/1998. Dabei galt es bei der Auswahl der einzelnen Gesellschaften zunächst zu beachten, daß in der deutschen Versicherungswirtschaft zwar zahlreiche Unternehmen die Rechtsform der Aktiengesellschaft aufweisen, allerdings sind nur wenige an einer Börse notiert. So wiesen von den 702 Versicherungsgesellschaften, die 1998 unter Bundesaufsicht standen, insgesamt

21) Ansätze zur Identifikation von Multifaktorenmodellen mit Hilfe der Zeitreihenregression bei in der Zeit variierenden Faktorladungen finden sich in Mayser 1996, S. 299-307.

22) Dabei wurde von den Autoren eine Portfolio-Bildung vorgenommen, um das „Fehler-in-denVariablen“-Problem zu kontrollieren. 
345 Gesellschaften die Rechtsform der Aktiengesellschaft auf, wobei nur 53 dieser Gesellschaften an einer Börse notiert waren. Dies ist vor allem auf die in der Versicherungswirtschaft weitverbreitete Konzernbildung und diese wiederum auf das aufsichtsrechtliche Gebot der Spartentrennung ( 8 Abs. 1a VAG) zurückzuführen. ${ }^{23)}$ Nach dem Spartentrennungsprinzip darf eine Versicherungsgesellschaft neben dem Lebens- bzw. Krankenversicherungsgeschäft keinen anderen Versicherungszweig betreiben. Insofern kann ein umfassendes Angebot an Versicherungsleistungen nur von mehreren Rechtseinheiten erbracht werden. Folglich sind die meisten Versicherungsaktiengesellschaften im 100\%-Besitz von Konzernen oder bilden durch Beteiligungen selber einen Konzern.

Von den vorliegenden börsennotierten Gesellschaften konnten nicht alle in die Untersuchung einbezogen werden. Zunächst blieben Rückversicherer unberücksichtigt, da sie sich in Geschäftsbetrieb und aufsichtsrechtlichen Rahmenbedingungen deutlich von Erstversicherern unterscheiden. ${ }^{24)}$ Weiterhin sind historisch bedingt für die einzelnen Versicherungsgesellschaften oftmals verschiedene Aktienarten (vinkulierte Namensaktien, Inhaberaktien) bzw. Aktiengattungen im Sinne des $\S 11$ AktG mit unterschiedlichen Nennwerten an der Börse notiert. ${ }^{25)}$ Dabei ist regelmäßig zu beobachten, daß die verschiedenen Aktien einer Gesellschaft eine stark gleichläufige Kurs- bzw. Renditeentwicklung aufweisen. Um eine zu starke Gewichtung einzelner Gesellschaften bzw. die Berücksichtigung redundanter Informationen $\mathrm{zu}$ vermeiden, wurde pro Gesellschaft nur eine Aktiengattung ausgewählt.

Schließlich wurde geprüft, ob eine ausreichende Marktliquidität bei den einzelnen Gesellschaften gegeben war. Eine solche Restriktion ist wichtig, da bei einer zu geringen Marktliquidität in den betreffenden Wertpapieren die Schätzergebnisse verzerrt werden können. Als Indikatoren für die Marktliquidität ${ }^{26)}$ wurden dabei Informationen über den Streubesitz ${ }^{27)}$ sowie die Anzahl der Monate ohne Kursveränderungen $^{28)}$ in den betreffenden Wertpapiere herangezogen. Bei der

23) Vgl. Farny 1983, S. 33.

24) Vgl. Mei/Saunders 1994.

25) Vgl. Steffen 1967, S. 67-68

26) Formale Ansätze zur Messung der Liquidität werden etwa von Oesterhelweg/Schiereck 1993 oder Kempf 1998 diskutiert. Allerdings ist keines dieser Maße unproblematisch und setzen außerdem Informationen (etwa den Bid-Ask-Spread) voraus, welche für die hier vorliegenden historischen Zeitreihen nicht ermittelt werden konnten.

27) Als Datenquelle zur Ermittlung der Streubesitzanteile dienten die Saling Aktienführer bzw. Unternehmensangaben.

28) Ähnlich wurde auch bei Harrington 1983, S. 590 und Cummins/Harrington 1988, S. 21 vorgegangen. 
konkreten Auswahl der in die einzelnen Teilperioden einbezogenen Aktien wurden keine feststehenden quantitativen Ausschlußkriterien herangezogen. Vielmehr war auf Basis der aufgeführten Kriterien (Transaktionsvolumen, Streubesitz) der Gesamteindruck der betreffenden Aktie maßgeblich. Insgesamt wurden die 23 in der folgenden Tabelle aufgeführten Gesellschaften selektiert.

Diese Anzahl spiegelt nur die Auswahl der Aktien wider. Über eine Verfügbarkeit der Daten bezüglich des gesamten Untersuchungszeitraums ist damit noch nichts gesagt, dies führt dazu, daß sich das Sample weiter verkleinert, je weiter man in der Zeit zurück geht. Dies hängt damit zusammen, daß viele Aktien erst in den letzten Jahren an die Börse gebracht worden sind. Die folgende Tabelle zeigt die untersuchten Gesellschaften sowie das Jahr der erstmaligen Berücksichtigung.

Tabelle 1: Liste der in den einzelnen Perioden einbezogenen Gesellschaften

\begin{tabular}{|c|c|c|c|}
\hline Nr. & Gesellschaft & WKN & Jahr \\
\hline 1. & Aachener und Münchener Leben & 845392 & 1993 \\
\hline 2. & Aachener und Münchener Versicherung & 841080 & 1981 \\
\hline 3. & Albingia Versicherungs-AG & 845700 & 1975 \\
\hline 4. & Allianz Leben & 840300 & 1975 \\
\hline 5. & Allianz Holding & 840400 & 1975 \\
\hline 6. & Alte Leipziger Versicherung & 842301 & 1993 \\
\hline 7. & AXA Colonia Konzern & 841000 & 1975 \\
\hline 8. & AXA Colonia Leben & 841230 & 1993 \\
\hline 9. & DBV-Winterthur Holding & 841690 & 1993 \\
\hline 10. & Gerling Konzern & 841895 & 1993 \\
\hline 11. & Hermes Kreditversicherung & 841902 & 1993 \\
\hline 12. & Mannheimer AG Holding & 842800 & 1975 \\
\hline 13. & Nordstern Allgemeine Versicherung & 843300 & 1975 \\
\hline 14 & Nordstern Leben & 843400 & 1981 \\
\hline 15. & Nürnberger Beteiligungs-AG & 843596 & 1993 \\
\hline 16. & Rheinland Holding & 841513 & 1993 \\
\hline 17. & Thuringia Versicherung & 844600 & 1975 \\
\hline 18. & Vereinte Versicherung & 840703 & 1975 \\
\hline 19. & Victoria Versicherung & 845300 & 1975 \\
\hline 20. & Volksfürsorge Holding & 840450 & 1993 \\
\hline 21. & Württembergische Leben & 840502 & 1993 \\
\hline 22. & Württembergische und Badische Versicherung & 845500 & 1993 \\
\hline 23. & Württembergische AG Versicherungsbeteiligungs-Gesellschaft & 845600 & 1995 \\
\hline
\end{tabular}

Von den $i=1, \ldots, n$ ausgewählten Versicherungsaktien wurden die monatlichen Risikoprämien folgendermaßen ermittelt. Definiert man $P_{i, t}$ als den um Ausschüttungen und Kapitalmaßnahmen bereinigten Preis der $i$-ten Versicherungsaktie zum Zeitpunkt $t$ und $r_{f, t}$ als die Rendite einer risikolosen 
Anlage von $t$ bis $t+1$ dann berechnet sich die Risikoprämie für den Monat $t$ gemäß:

$$
R P_{i, t}=\frac{P_{i, t+1}-P_{i, t}}{P_{i, t}}-r_{f, t}
$$

Die bereinigten Kurse der Versicherungsaktien stammen von der Deutschen Finanzmarktdatenbank in Karlsruhe bzw. von Datastream. Für den risikolosen Zinssatz wurde der jeweils am Monatsanfang vorliegende Frankfurter Interbankenzins (FIBOR) verwendet. ${ }^{29)}$ Die nachfolgende Tabelle enthält für jedes Jahr im Zeitraum 1975 bis 1998 die durchschnittliche Risikoprämie der $n$ einbezogenen Einzelwerte, deren Streuung sowie den kleinsten und größten Wert. Dabei ist zu erkennen, daß die beobachteten Risikoprämien sowohl zwischen verschiedenen Gesellschaften in einer Periode sowie zwischen verschiedenen Perioden erheblichen Schwankungen unterlagen.

Tabelle 2: Risikoprämien deutscher Versicherungsaktien (in \% p.a.)

\begin{tabular}{rrrrrr}
\hline Jahr & MW & STD & Min & Max & n \\
\hline 1975 & 4,45 & 16,96 & $-15,26$ & 30,76 & 9 \\
1976 & $-10,75$ & 18,88 & $-43,47$ & 17,58 & 9 \\
1977 & 7,25 & 12,29 & $-9,00$ & 30,26 & 9 \\
1978 & 33,30 & 48,14 & 1,34 & 154,52 & 9 \\
1979 & $-1,48$ & 16,39 & $-34,06$ & 28,79 & 9 \\
1980 & $-1,91$ & 17,75 & $-27,71$ & 28,50 & 9 \\
1981 & $-19,12$ & 23,85 & $-41,90$ & 17,11 & 11 \\
1982 & 20,59 & 25,06 & $-12,69$ & 74,56 & 11 \\
1983 & 33,77 & 17,02 & 6,68 & 56,69 & 11 \\
1984 & 27,22 & 31,58 & $-4,23$ & 102,42 & 11 \\
1985 & 95,77 & 54,24 & 48,09 & 211,98 & 11 \\
1986 & $-1,86$ & 27,69 & $-44,70$ & 31,41 & 11 \\
1987 & $-48,61$ & 14,84 & $-74,27$ & $-27,97$ & 11 \\
1988 & 5,93 & 5,79 & $-2,07$ & 14,28 & 11 \\
1989 & 38,83 & 29,65 & $-25,56$ & 92,40 & 11 \\
1990 & $-14,32$ & 25,69 & $-54,69$ & 39,92 & 11 \\
1991 & $-4,74$ & 6,34 & $-15,62$ & 8,64 & 11 \\
1992 & $-26,76$ & 14,89 & $-53,17$ & 2,86 & 11 \\
1993 & 30,85 & 21,18 & $-26,78$ & 69,05 & 22 \\
1994 & $-17,50$ & 19,31 & $-42,58$ & 29,95 & 22 \\
1995 & $-2,36$ & 4,67 & $-15,35$ & 7,36 & 23 \\
1996 & 2,79 & 11,22 & $-16,64$ & 23,13 & 23 \\
1997 & 31,27 & 26,05 & $-17,11$ & 72,57 & 23 \\
1998 & 11,81 & 19,50 & $-25,69$ & 58,32 & 23 \\
\hline
\end{tabular}

Erläuterung: "MW" bezeichnet den arithmetischen Mittelwert der Risikoprämien der $n$ im betreffenden Jahr einbezogenen Versicherungsaktien, „STD“ die zugehörige Standardabweichung, „Min“ den kleinsten und „Max“ den größten Wert.

29) Die Umrechnung von per annum quotierten FIBOR-Sätzen $r_{f, p . a .}$ auf Monatsbasis erfolgte gemäß $r_{f, p . m .}=\left(1+r_{f, p . a .}\right)^{1 / 12}$. 


\subsection{Auswahl der Faktoren}

Im Kontext der hier betrachteten Längsschnittanalysen kommt der Auswahl der Variablen, welche die Marktrisikoprämien der Versicherungsaktien erklären sollen, eine zentrale Bedeutung zu. Dabei ist es zum einen wichtig möglichst viele der Faktoren, die einen ökonomischen Einfluß auf die Aktienkursentwicklung haben, zu berücksichtigen. Andererseits ist bei der Modellspezifikation das Prinzip der sparsamen Parametrisierung zu beachten. Um zwischen diesen beiden konkurrierenden Zielen einen sinnvollen Kompromiß zu finden, wurde bei der Auswahl der Faktoren für diese Studie auf die folgenden Kriterien geachtet: Welche Faktoren erscheinen ökonomisch plausibel? Welche Faktoren wurden bei ähnlichen Studien für signifikant befunden? In Anlehnung an die Studie von Mei/Saunders (1994) für US-amerikanische Versicherungsaktien wurden die folgenden sechs Faktoren ausgewählt ${ }^{30)}$ :

(1) Allgemeines Zinsniveau: Die in Theorie und Praxis angewendeten Verfahren zu Unternehmens- bzw. Aktienbewertung enthalten regelmäßig eine Zinskomponente zur Diskontierung zukünftiger Cash-Flows. Insofern sollte das allgemeine Zinsniveau eine der wichtigsten Determinanten zur Erklärung von Aktienkursentwicklungen darstellen. Das gilt für Aktien allgemein, aber insbesondere auch für Versicherungsaktien, die aufgrund ihrer Geschäftsstruktur als zinssensitiv gelten. So wirken Veränderungen des Zinsniveaus sowohl auf das versicherungstechnische Ergebnis (etwa beim Cash-Flow-Underwriting) als auch auf das Ergebnis aus den Vermögensanlagen. Als Indikator für das aktuelle Niveau der Zinsstrukturkurve wurde die Rendite für zehnjährige deutsche Bundesanleihen verwendet. $^{31)}$

(2) Form der Zinsstrukturkurve: Die Form der Zinsstrukturkurve wird hier durch die Differenz von lang- und kurzfristigen Zinsen quantifiziert. Dabei deutet ein positiver (negativer) Spread zwischen lang- und kurzfristigen Zinsen auf eine normale (inverse) und ein Zinsspread von null auf eine flache Zinsstruktur hin. Als Repräsentant für langfristige Zinsanlagen dient die

30) Die Quelle für die makroökonomischen Daten ist Datastream.

31) Als Alternative zur Messung des allgemeinen Zinsniveau könnten kurzfristige Geldmarktzinsen, anstatt langfristiger Renditen von Staatsanleihen verwendet werden, vgl. etwa Mei/Saunders 1994. Aufgrund der hohen positiven Korrelation zwischen lang- und kurzfristigen Zinsen werden die Schätzergebnisse dieser beiden Varianten weitgehend miteinander korrespondieren. Die Wahl auf die langfristigen Zinsen in dieser Studie begründet sich, weil die kurzfristigen Zinsen eine deutlich höhere Korrelation mit den anderen Erklärungsvariable (insbesondere dem Zinsspread) aufwiesen. 
Umlaufrendite für zehnjährige Bundesanleihen, wogegen für kurzfristige Zinstitel der dreimonatige FIBOR verwendet wurde. Für die zinssensitiv geltenden Versicherungsaktien könnte diese Variable von besonderer Bedeutung $\mathrm{zu}$ sein, da der Zinsspread auch Informationen über die Erwartungen der Marktteilnehmer bezüglich der zukünftigen Zinsentwicklung enthält.

(3) Der Wechselkurs der Deutschen Mark zum US-Dollar: Als erklärende Variable wird die monatliche relative Veränderung des Wechselkurses der DM zum US-Dollar herangezogen. Hierbei handelt es sich um eine Variable, die in anderen Studien eher fehlt. Dies liegt daran, daß sich der größte Teil der hier angeführten Arbeiten auf den amerikanischen Markt bezieht. Der Wechselkurs gegenüber der DM spielt dort eine weitgehend untergeordnete Rolle. Für den deutschen Aktienmarkt läßt sich jedoch eine signifikante Korrelation mit der Kursentwicklung des US-Dollars gegenüber der DM beobachten. Dies hängt einerseits mit der Leitfunktion der New Yorker Börse zusammen, hat andererseits aber auch fundamentale Gründe, da die gesamte deutsche Wirtschaft stark vom Außenhandel abhängig ist, wobei im allgemeinen in US-Dollar fakturiert wird. ${ }^{32)}$

(4) Unerwartete Inflation: Diese Variable findet man auch in den meisten der oben zitierten Studien. ${ }^{33)}$ Gerade für Versicherungsaktien scheint sie auch von Bedeutung zu sein, was sich daraus ableitet, daß die meisten Zahlungsströme durch den Nominalzins determiniert sind. Es ist wichtig, darauf hinzuweisen, daß hierbei die unerwartete Inflation von Interesse ist. Die erwartete und tatsächliche Inflation der Vorperioden sind als a priori Information schon in den Kursen enthalten, so daß nur noch die unerwartete Inflation in einer gegebenen Periode die Aktienkurse beeinflussen kann. Eine bedeutsame Problematik ist, daß die von den Marktteilnehmern erwartete Inflationsrate keine beobachtbare Größe darstellt. Sie muß daher in geeigneter und transparenter Weise modelliert werden. Hierzu wurde auf ein von

32) Vgl. hierzu die Studie von Entorf 2000. Hierbei wird auf der Basis rollierender Regressionen und Kointegrationsanalysen festgestellt, daß zwischen dem Deutschen Aktienindex (DAX) und dem Wechselkurs zum US-Dollar im Untersuchungszeitraum 1974-1995 eine statistisch signifikante Beziehung besteht.

33) Chen et al. 1986 messen der Inflation eine besonders hohe Bedeutung zu, sie verwenden die Änderung der erwarteten Inflation und die unerwartete Inflation. Jedoch finden die Autoren, daß die Signifikanz der Inflation für Aktienrenditen hinter der der anderen makroökonomischen Variablen zurückbleibt. Vgl. Chen et al. 1986, S. 396, S. 398 und S. 402. 
Maurer/Eberts (2000) vorgeschlagenes ARMA(1,1)-Zeitreihenmodell ${ }^{34)}$ zurückgegriffen, welches die Entwicklung der Inflationsrate gemäß der Gleichung

$$
I_{t}=0,0026+0,98 I_{t-1}+U_{t}-0,87 U_{t-1}
$$

abbildet. Dabei bezeichnet $I$ die Inflationrate berechnet auf Basis des von der Deutschen Bundesbank nach dem modifizierten Census X-11 Verfahren saisonbereinigten Preisindex für die Lebenshaltung aller privaten Haushalte im früheren Bundesgebiet $(1991=100), U$ einen Zufallsterm und $t$ den Zeitparameter. Mit Hilfe der auf den Zeitpunkt $t-1$ konditionierten Informationen kann die erwartete Inflationrate für die nächste Periode mit Hilfe von Gleichung (4) durch Ein-Schrittprognose ermittelt werden. Die unerwartete Inflationsrate ergibt sich als Differenz aus der zu Beginn der Periode erwarteten und der am Periodenende realisierten Inflationsrate.

(4) Veränderungen der realen Industrieproduktion: Diese Variable wird verwendet, um den Einfluß der allgemeinen wirtschaftlichen Entwicklung auf die Veränderung von Aktienkursen abzubilden. ${ }^{35)}$ Ökonomisch scheint das wirtschaftliche Wachstum vor allem für die Kompositversicherer von Bedeutung zu sein, da diese in einem eher zyklischeren Geschäftsumfeld arbeiten als die Anbieter von Lebensversicherungen. ${ }^{36)}$

(5) Der Januareffekt: Dieser wird hier durch eine Dummyvariable modelliert, die für den Monat Januar den Wert eins und sonst den Wert null annimmt. Ein möglicher Erklärungsansatz des Januareffektes ist das Phänomen, daß viele Anleger, insbesondere Fonds, zu Beginn des Jahres in großem Umfang Aktien kaufen, was die Kurse meist in die Höhe treibt. ${ }^{37)}$ Es ist also zu erwarten, daß

34) Im Rahmen der vorliegenden Untersuchung wurde auch versucht, die erwartete Inflation durch die Inflation der Vorperiode zu modellieren, was stationären Erwartungen der Marktteilnehmer entspricht. Somit ergäbe sich die unerwartete Inflation als Differenz der aktuellen Inflation und der Inflation der vorangegangenen Periode. Die Ergebnisse dieser Modellvariante unterscheiden sich nur unwesentlich von der im folgenden vorgestellten.

35) Als Alternative bietet sich das Bruttosozialprodukt an, welches allerdings nur quartalsweise zur Verfügung stand. In dieser Studie wurde der Industrieproduktion der Vorrang gegeben. Beide Variablen zu verwenden ist statistisch problematisch, da sie naturgemäß stark miteinander korreliert sind. Schließlich stammt ein großer Teil des Bruttosozialprodukts aus der Industrieproduktion. Der Korrelationskoeffizient dieser beiden Variablen beträgt für den gesamten Untersuchungszeitraum 0,8081.

36) Vgl. GDV 1997, S. 6.

37) Die Berücksichtigung des Januareffekts in vergleichbaren empirischen Studien ist uneinheitlich. So berücksichtigen Roll/Ross 1995, Burmeister et al. 1994 und Connor 1995 ihn nicht, wohingegen Mei/Saunders 1994 diesen Dummy verwenden, allerdings nicht mit 
die Aktienrenditen im Januar signifikant höher sind als in den übrigen Monaten eines Jahres.

Nachdem die in der vorliegenden Studie verwendeten erklärenden Variablen beschrieben worden sind, ist es geboten, zu erläutern, warum einige bekannte Variablen, die üblicherweise herangezogen werden, hier keine Berücksichtigung fanden. Dabei geht es vor allem um den corporate credit spread und die Dividendenrendite des Gesamtmarktes. ${ }^{38)}$

Der corporate credit spread, definiert als Differenz zwischen der Rendite von Unternehmens- und Staatsanleihen, ist ein Maß für das Vertrauen, das aktuell in die Volkswirtschaft gesetzt wird. Diese Größe kann interpretiert werden als die (Kredit-)Risikoprämie, die Unternehmen bei der Aufnahme von Fremdkapital zahlen müssen. ${ }^{39)}$ Allerdings war diese Größe für den Untersuchungszeitraum praktisch nicht verfügbar, da sich der Markt für Unternehmensanleihen in Deutschland gerade erst entwickelt.

Die allgemeine Dividendenrendite kann als Indikator für das Bewertungsniveau eines Aktienmarktes gesehen werden. Für eine Studie über den deutschen Markt, wie die hier vorliegende, erscheint sie aber als erklärende Variable im Gegensatz zum amerikanischen Aktienmarkt aus zwei Gründen wenig zweckmäßig: Erstens wird in Deutschland die Dividende nur einmal im Jahr gezahlt, und zweitens ist der Informationsgehalt einer deutschen Dividendenzahlung an sich eher gering. Das liegt daran, daß sie meist losgelöst von der Entwicklung des Geschäftsjahres ist und vielmehr mehr oder weniger stetig im Zeitablauf steigt.

Eine andere erklärende Variable, die in diese Studie nicht mit einbezogen wurde, ist die Rendite eines geeigneten Index für den Gesamtmarkt. Eine Einbeziehung derselben wird in der Literatur kontrovers diskutiert; in manchen Studien fehlt sie ganz ${ }^{40)}$ andere argumentieren, daß Multifaktorenmodelle eine Erweiterung des CAPM seien und verwenden sie dementsprechend. ${ }^{41)}$ In der vorliegenden Arbeit wurde diese Variable nicht verwendet, da es im Rahmen der APT auch möglich

signifikanten Ergebnissen. Zu einer weiterführenden Behandlung des Januareffektes, vgl. etwa Rozeff/Kinney 1979 oder Haugen 1997, S. 678-692.

38) Vgl. etwa Mei/Saunders 1994.

39) Vgl. etwa Burmeister et al. 1994. S. 316 oder Chen et al. 1986, S. 389 zur Motivation des corporate credit spreads.

40) So etwa in Connor 1995.

41) Wei 1998 stellt ein allgemeines Preisbildungsmodel vor, welches das CAPM und die APT kombiniert. Burmeister et al. 1994, S. 317 benutzen nur den Teil des Gesamtmarktes, der nicht bereits durch die anderen Faktoren erklärt wird und definieren dies als market-timing risk. 
sein muß, die Rendite des Gesamtmarktes mit Hilfe der benutzten Risikofaktoren zu erklären. Folglich sollte die Leistung eines Multifaktorenmodells gerade darin bestehen, die Renditen der Finanztitel zu beschreiben, ohne auf den Gesamtmarkt zurückgreifen zu müssen. ${ }^{42)}$

Die nachfolgende Tabelle 3 enthält die Mittelwerte, Standardabweichungen sowie die entsprechenden Korrelationen der einbezogenen Variablen, berechnet für den gesamten Untersuchungszeitraum:

Tabelle 3: Korrelationsmatrix der verwendeten Faktoren im Zeitraum 1975-1998

\begin{tabular}{cccccccc}
\hline & & & \multicolumn{5}{c}{ Korrelationen } \\
\cline { 3 - 8 } & & STD & Zinsniveau & Zinsspread & USD & $\begin{array}{c}\text { Unerw. } \\
\text { Inflation }\end{array}$ & IP \\
\hline Zinsniveau & 7,30 & 1,29 & 1 & & & & \\
Zinsspread & 1,20 & 1,70 & $-0,3555$ & 1 & & & \\
$\quad$ USD & $-0,07$ & 3,32 & 0,0892 & $-0,0293$ & 1 & & \\
Unerw. Inflation & 0,12 & 0,49 & $-0,0459$ & 0,0442 & 0,1110 & 1 & \\
IP & 1,41 & 4,29 & $-0,1523$ & 0,2067 & $-0,1264$ & 0,0173 & 1 \\
\hline
\end{tabular}

Erläuterungen: „Zinsniveau“ bezeichnet die annualisierte Umlaufrendite 10jähriger Bundesanleihen (\% p.a.); „Spread“ die Differenz zwischen der Umlaufrendite 10jähiger Bundesanleihen und dem 3-Monatsgeldmarktzins (in \% p.a.); „Unerw. Inflation“ die unerwartete Inflationsrate; „USD“ die relative Veränderung des US-Dollars zur DM (\% p.m.); „IP“ das Wachstum der Industrieproduktion; „MW“ steht für den Mittelwert und „STD“ für die Standardabweichung der jeweiligen Variablen.

Man erkennt, daß im Betrachtungszeitraum die ausgewählten Faktoren nicht alle vollständig unkorreliert und damit auch nicht (stochastisch) unabhängig sind. Während sich das Ausmaß der Korrelationen bei den meisten Variablen in einem beschränkten Rahmen halten, ist der Zusammenhang zwischen den Renditen langfristiger Bundesanleihen und dem Zinsspread ausgeprägt. Ein Verfahren um den daraus resultierenden statistischen Problemen aus der Multikolinearität von erklärenden Variablen Rechnung $\mathrm{zu}$ tragen, besteht in der Vornahme einer Orthogonalisierung. Dies hätte allerdings den Nachteil, daß die Faktoren ökonomisch nur eingeschränkt interpretierbar wären. Im weiteren sollen daher nur die Ergebnisse der nicht bereinigten Faktoren vorgestellt werden, da sie ökonomisch aussagekräftiger sind.

42) Dies ist im übrigen auch empirisch beobachtbar. Die Bedeutung des Marktindexes zur Erklärung der Aktienrenditen ist zwar vorhanden, benutzt man jedoch zusätzlich auch makroökonomische Variablen, so zeigt sich, daß der Index nahezu unbedeutend wird. Vgl. hierzu Chen et al. 1986, S. 395ff. 


\section{Empirische Ergebnisse}

\subsection{Erklärungskraft des Modells}

Der Untersuchungszeitraum von 1975 bis 1998 (jeweils einschließlich) umfaßt 24 Jahre, welche in vier nicht überlappende Subperioden à sechs Jahre (bzw. 72 Monaten) - Periode 1: 01/1975-12/1980, Periode 2: 01/1981-12/1986, Periode 3: 01/1987-12/1992 und Periode 4: 01/1993-12/1998 - zerlegt wurden. Die Bildung von Teilperioden bei der empirischen Modellspezifikation ist zweckmäßig, da sich der Einfluß von makroökonomischen Risikofaktoren auf die Aktienrenditen im Zeitablauf durchaus ändern kann. Anderseits ist eine gewisse Periodenlänge erforderlich, um eine ausreichende Präzision von statistischen Inferenzaussagen zu gewährleisten. Dabei hat sich in vergleichbaren Studien ein Zeitintervall von fünf bis sechs Jahren als sinnvoll herausgestellt. ${ }^{43)}$

Für jede Periode wurde dann für jede Aktie und für einen gleichgewichteten Index eine lineare Regression durchgeführt, wobei die realisierte Risikoprämie mit den Faktorausprägungen des dazugehörigen Monats erklärt wurde. Nachfolgende Tabelle 4 zeigt die Erklärungskraft anhand des statistischen Bestimmtheitsmaßes $\left(\mathrm{R}^{2}\right)$. Das $\mathrm{R}^{2}$ gibt an, welcher Teil der Varianz der zu erklärenden Variablen mit den selektierten Regressoren erklärt werden kann.

Tabelle 4: Erklärungskraft des Modells

\begin{tabular}{lcccc}
\hline & $\mathbf{1 9 7 5 - 1 9 8 0}$ & $\mathbf{1 9 8 1 - 1 9 8 6}$ & $\mathbf{1 9 8 7 - 1 9 9 2}$ & $\mathbf{1 9 9 3 - 1 9 9 8}$ \\
\hline Anzahl der Aktien & 9 & 11 & 11 & 23 \\
Mittleres R $^{2}$ & $13,62 \%$ & $14,12 \%$ & $9,29 \%$ & $9,62 \%$ \\
Größtes R $^{2}$ & $21,82 \%$ & $28,97 \%$ & $16,21 \%$ & $21,06 \%$ \\
Kleinstes R $^{2}$ & $5,88 \%$ & $5,94 \%$ & $1,24 \%$ & $2,03 \%$ \\
$\mathrm{R}^{2}$ des Indexes & $19,29 \%$ & $11,61 \%$ & $13,03 \%$ & $14,66 \%$ \\
Mittleres R $^{2}$ der fünf & $18,64 \%$ & $12,80 \%$ & $12,64 \%$ & $14,67 \%$ \\
liquidesten Werte & & & & \\
\hline
\end{tabular}

Erläuterungen: Die unterschiedliche Anzahl der untersuchten Aktien in den Subperioden liegt an der Verfügbarkeit der Daten und ist dementsprechend in den frühen Perioden kleiner. Der Index ist ein gleichgewichtetes Portefeuille aus allen Aktien, für die Kursdaten zur Verfügung standen. Die fünf liquidesten Werte sind Allianz Leben, Allianz Holding, AXA Colonia, Mannheimer und Nordstern Allgemeine. Diese Auswahl ist in allen vier Perioden konstant gehalten.

Es wird deutlich, daß die verwendeten makroökonomischen Faktoren die Renditeschwankungen von Aktien nur zum Teil erklären können. Die hier erzielten Ergebnisse liegen im Rahmen vergleichbarer Studien. So kommt Connor (1995, S. 45) auf ein $\mathrm{R}^{2}$ von 10,9\% für Aktien allgemein und Mei/Saunders (1994, S. 20) auf ein adjustiertes $R^{2}$ von $5,2 \%$ für einen Index aus Kompositversicherern

43) Vgl. Mei/Saunders 1994 oder Cummins/Harrington 1988. 
und 6,4\% für einen Index aus Lebensversicherern. Dies entspricht in dem Kontext dieser Studie (72 Perioden bei 6 Regressoren) einem nicht-adjusitierten $\mathrm{R}^{2}$ von $13,2 \%$ und $14,3 \%$ respektive. Bemerkenswert ist, daß die liquiden Werte im allgemeinen besser durch das Modell erklärt werden. Dies ist nicht überraschend, da eine vernünftige Funktionsweise der Faktorenmodelle nur bei hinreichender Liquidität der zugrundeliegenden Finanzinstrumente gewährleistet werden kann. Dieses Phänomen spiegelt sich auch in der Tatsache wider, daß die Ergebnisse für den Index aus allen Versicherungsaktien deutlich besser sind. Einflüsse, die nur einzelne Aktien betreffen, werden hier offensichtlich über alle Aktien weitgehend nivelliert, so daß die Erklärungskraft des Modells steigt.

Um einen graphischen Eindruck von der Höhe und den Schwankungen der tatsächlichen bzw. erwarteten Risikoprämien zu vermitteln, sind in der folgenden Abbildung die entsprechenden Werte für den betrachteten Index aus Versicherungsaktien im Zeitraum 1/1975-12/1998 abgetragen.

Abbildung 1: Erwartete bzw. realisierte Risikoprämien für den Index

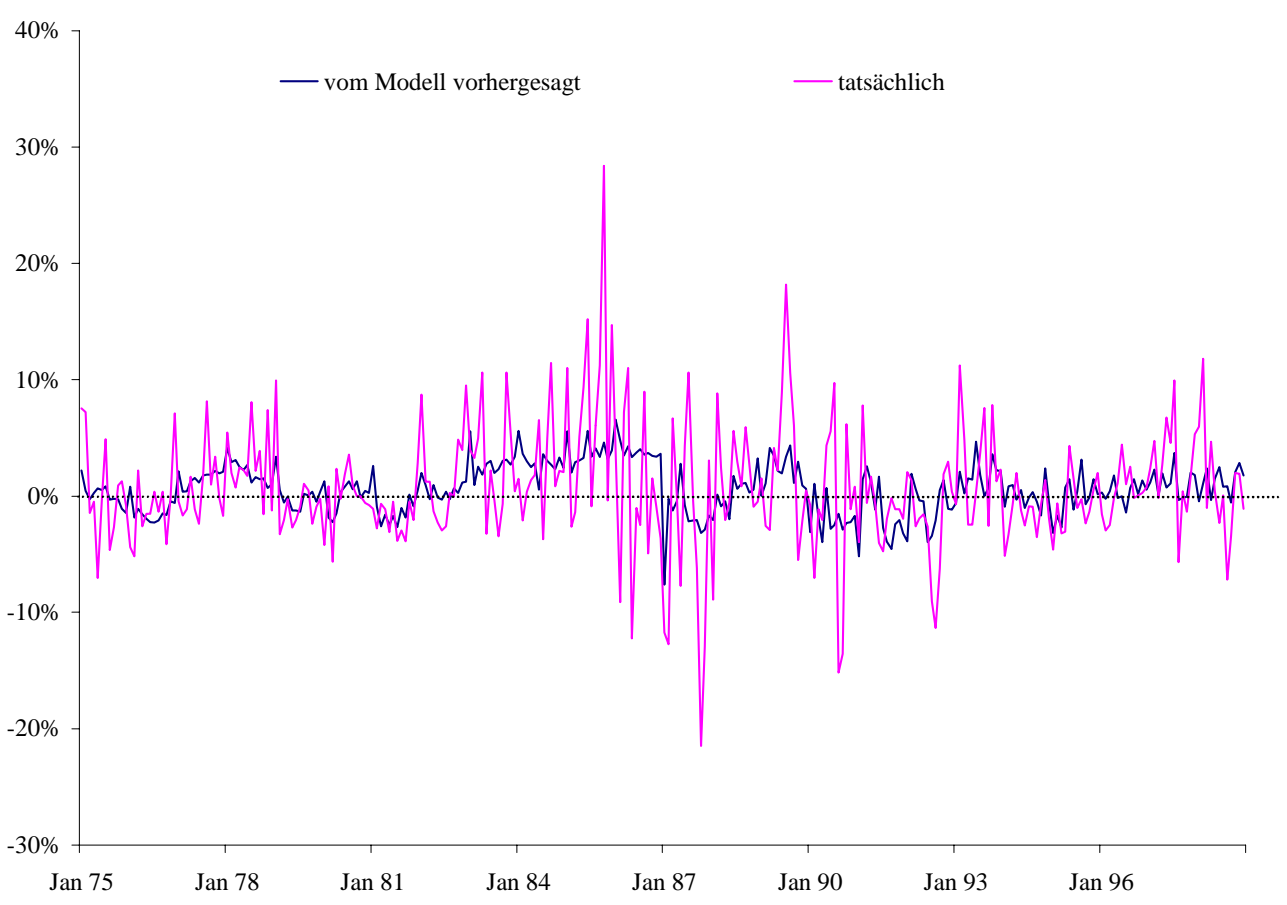

Aus der Abbildung ist $\mathrm{zu}$ erkennen, dass die vom Modell errechneten Risikoprämien im Zeitablauf wenig stabil sind. Dies resultiert daraus, dass die verwendeten makroökonomischen Faktoren im Zeitablauf ebenso deutlichen Schwankungen unterlagen, was sich auf die Modell ermittelten erwarteten Risikoprämien durchschlägt. Allerdings wird aus der Abbildung ebenfalls deutlich, dass die erwarteten Risikoprämien im Zeitablauf deutlich geringeren 
Schwankungen unterlagen, als die tatsächlich realisierten Werte. So kann Tabelle 5 entnommen werden, daß etwa im Zeitraum 1975-1980 die Volatilität der realisierten $(3,43 \%)$ im Vergleich zu den erwarteten $(1,51 \%)$ Risikoprämien mehr als doppelt so hoch ausfiel. Ähnliches gilt für die anderen Teilperioden.

Tabelle 5: Erwartete bzw. realisierte Risikoprämien für den Index (in \% p.m.)

\begin{tabular}{lcccccccc}
\hline Zeitraum & \multicolumn{2}{c}{ MW } & \multicolumn{2}{c}{ STD } & \multicolumn{2}{c}{ Min } & \multicolumn{2}{c}{ Max } \\
\hline $1975-1980$ & 0,40 & $(0,40)$ & 1,51 & $(3,43)$ & $-2,26$ & $(-7,02)$ & 4,14 & $(9,92)$ \\
$1981-1986$ & 2,03 & $(1,97)$ & 2,40 & $(6,38)$ & $-7,62$ & $(-12,24)$ & 6,59 & $(28,36)$ \\
$1987-1992$ & $-0,50$ & $(-0,32)$ & 2,26 & $(6,62)$ & $-5,17$ & $(-21,47)$ & 4,35 & $(18,16)$ \\
$1993-1998$ & 0,71 & $(0,58)$ & 1,44 & $(3,59)$ & $-3,17$ & $(-7,17)$ & 4,69 & $(11,82)$ \\
\hline
\end{tabular}

Erläuterungen: „MW“ bezeichnet die durchschnittliche erwartete Risikoprämie, „STD“ die zugehörige Standardabweichung, „Min“ den kleinsten und „Max“ den größten Beobachtungswert im jeweiligen Betrachtungszeitraum. In Klammern sind die korrespondierenden Werte für die realisierten Risikoprämien angegeben.

Eine weitere interessante Beobachtung ist, daß in einzelnen Perioden die erwarteten Risikoprämien negativ ausfallen. ${ }^{44)}$ Eine mögliche Erklärung könnte darin liegen, daß Versicherungsaktien in bestimmten ökonomischen Situationen gute Hedge-Eigenschaften gegenüber ausgewählten Risikofaktoren aufweisen. Eine weitere Erklärung könnte in Überreaktionen der Marktteilnehmer liegen. So könnten die Investoren in bestimmten Börsenphasen $\mathrm{zu}$ optimistisch hinsichtlich der zukünftigen Wertentwicklung von Versicherungsaktien gewesen sein und deshalb derart hohe Preise bereit waren zu zahlen, so daß die Risikoprämien negativ ausfielen. Dies tritt besonders deutlich für den Zeitraum von 1987 bis 1992 zum Vorschein, indem sich eine durchschnittlich negative erwartete Risikoprämie von -0,50\% ergab. Dabei waren in den Börsenjahren 1987 und 1990 die erwarteten Risikoprämien mit Ausnahme weniger Monate stets negativ. Der Spitzenwert ergab sich im Januar 1987 mit -7,621\%. Die nachfolgenden Börsencrashs in diesen Jahren führten zu einer (drastischen) Kurskorrektur und den Erwartungen der Marktteilnehmer.

\subsection{Bedeutung einzelner Faktoren}

Neben der Erklärungskraft des Modells ist es von Interesse, inwieweit einzelnen Faktoren ein signifikanter Einfluß auf die Aktienkursentwicklungen nachgewiesen werden kann. Hierzu wird ein t-Test bezüglich den Schätzwerten für die

44) Auch Mei/Saunders 1994, S. 22-23 berichten negative erwartete Risikoprämien. 
Regressionskoeffizienten durchgeführt. Die folgende Tabelle zeigt dessen Ergebnisse, zunächst für einzelne Aktien, auf:

Tabelle 6: Bedeutung der Faktoren für einzelne Aktien

\begin{tabular}{lcccc}
\hline & $\mathbf{1 9 7 5 - 1 9 8 0}$ & $\mathbf{1 9 8 1 - 1 9 8 6}$ & $\mathbf{1 9 8 7 - 1 9 9 2}$ & $\mathbf{1 9 9 3 - 1 9 9 8}$ \\
\hline Anzahl der Aktien & 9 & 11 & 11 & 23 \\
Konstante & $0,1002(4 / 3)$ & $0,0606(1 / 0)$ & $0,1580(4 / 1)$ & $0,0376(0 / 0)$ \\
10jähriger Zins & $-1,2072(4 / 3)$ & $-0,6024(1 / 0)$ & $-2,2531(4 / 2)$ & $-0,3775(1 / 0)$ \\
Zinsspread & $-0,2026(0 / 0)$ & $0,4596(1 / 0)$ & $-1,3370(1 / 0)$ & $-0,3783(2 / 2)$ \\
Veränderung des USD & $-0,0537(4 / 2)$ & $-0,0454(0 / 0)$ & $0,3055(2 / 1)$ & $0,4624(12 / 8)$ \\
Unerwartete Inflation & $0,0056(0 / 0)$ & $0,0109(2 / 2)$ & $-0,0064(0 / 0)$ & $0,0012(1 / 0)$ \\
Veränderung der IP & $-0,0017(3 / 3)$ & $0,0016(1 / 1)$ & $0,0053(5 / 2)$ & $0,0000(0 / 0)$ \\
Januardummy & $0,0207(1 / 1)$ & $0,0296(2 / 2)$ & $-0,0430(1 / 0)$ & $-0,0244(4 / 0)$ \\
\hline
\end{tabular}

Erläuterungen: Die angegebenen Werte entsprechen jeweils dem Mittelwert der Koeffizientenschätzung über alle einbezogenen Aktien. In Klammern ist die Anzahl der Aktien angegeben, für die dieser Faktor signifikant zum 10\%-Niveau von Null verschieden ist, der zweite Wert gibt das gleiche für das 5\%-Niveau an. USD steht für US-Dollar und IP für Industrieproduktion.

Die folgende Tabelle zeigt die Ergebnisse der Koeffizientenschätzungen für den gleichgewichteten Index der einbezogenen Versicherungsaktien.

Tabelle 7: Bedeutung der Faktoren für den Versicherungsindex

\begin{tabular}{lcccc}
\hline & $\mathbf{1 9 7 5 - 1 9 8 0}$ & $\mathbf{1 9 8 1 - 1 9 8 6}$ & $\mathbf{1 9 8 7 - 1 9 9 2}$ & $\mathbf{1 9 9 3 - 1 9 9 8}$ \\
\hline Konstante & $0,098 * *(3,22)$ & $0,0606(0,819)$ & $0,151 *(1,80)$ & $0,038(1,24)$ \\
10jähriger Zins & $-1,21 * *(-3,08)$ & $-0,602(-0,707)$ & $-2,17 *(-1,93)$ & $-0,382(-0,750)$ \\
Zinsspread & $-0,176(-0,874)$ & $0,459(0,6497)$ & $-1,21(-1,63)$ & $-0,381(-0,912)$ \\
Veränderung des USD & $-0,0379(-0,284)$ & $-0,0454(-0,212)$ & $0,307(1,46)$ & $0,464 * *(2,82)$ \\
Unerwartete Inflation & $0,0046(0,556)$ & $0,0109(0,634)$ & $-0,008(-0,564)$ & $0,0013(0,164)$ \\
Veränderung der IP & $-0,0017 * *(-2,22)$ & $0,0016(0,790)$ & $0,0056 *(2,02)$ & $0,0000(0,0354)$ \\
Januardummy & $0,0217(1,5780)$ & $0,0296(1,09)$ & $-0,043(-1,54)$ & $-0,0240(-1,51)$ \\
\hline
\end{tabular}

Erläuterungen: Die Zahlen sind jeweils die Koeffizientenschätzung für den Index. In Klammern ist der t-Wert angegeben. Die mit ,"“ bzw. mit ,**“ gekennzeichneten Werte sind signifikant zum 10\%- bzw. 5\%-Niveau. USD steht für US-Dollar und IP für Industrieproduktion.

Bei der Betrachtung dieser Ergebnisse zeigt sich, daß wie erwartet Veränderungen des allgemeinen Zinsniveaus einen substantiellen Einfluß auf die Risikoprämien von Versicherungsaktien haben. Dabei ist der zugehörige Koeffizient in allen vier Unterperioden negativ, d.h. Zinssteigerungen gehen in der Tendenz mit fallenden Risikoprämien einher. Für eine Reihe von Gesellschaften ist dieser Einfluß signifikant und für den Index läßt sich in zwei der vier Unterperioden eine deutliche Signifikanz feststellen. Die Bedeutung des langfristigen Zinses für die Versicherungsaktien scheint in der vierten Periode abzunehmen. Dies kann darauf zurückzuführen sein, daß es in dieser Periode zwei durch Börsencrashs ausgelöste lang anhaltende Baissephasen (im Sommer 1997 und Herbst 1998) gab, die zu einer kurzfristigen Inversion des klassischen Zinsverhaltens vieler Sektoren so 
auch der Versicherungen führten. ${ }^{45)}$ Insgesamt korrespondieren die Ergebnisse mit denen von Mei/Saunders, die für die Risikoprämien eines Index aus USamerikanischer Versicherungsaktien ebenfalls eine signifikant negative Abhängigkeit vom allgemeinen Zinsniveau feststellen konnten. ${ }^{46)}$

Im Vergleich zum allgemeinen Zinsniveau fällt der Einfluß des Zinsspreads, als Indikator für die Form der Zinsstrukturkurve, deutlich weniger sensitiv und signifikant aus. Für den Index ist der entsprechende Koeffizient in keiner Teilperiode auf dem 10\%-Niveau signifikant von Null verschieden. Auch für die Einzelwerte lassen sich nur für wenige Gesellschaften signifikante Ergebnisse beobachten. Auch diese Ergebnisse sind konsistent mit den empirischen Befunden von Mei/Saunders.

Der Einfluß von Wechselkursveränderungen gegenüber dem US-Dollar auf die Risikoprämien deutscher Versicherungsaktien war in den beiden ersten Perioden negativ, während sich in der dritten und vierten Teilperiode das Vorzeichen umkehrt. $^{47)}$ Dabei ist $\mathrm{zu}$ beobachten, daß die Sensitivität gegenüber Wechselkursschwankungen beständig zunimmt. So führte etwa im Beobachtungszeitraum 1975-1980 (1993-1998) eine Dollarsteigerung um 1\% zu einem durchschnittlichen Rückgang (Zunahme) der Risikoprämie des gleichgewichteten Index aus Versicherungsaktien um 0,0379\% (0,464\%). Des weiteren steigt die Signifikanz des Einflusses von Wechselkursschwankungen. So ist der Koeffizient in der vierten Periode für zwölf der 23 Gesellschaften signifikant zum 10\%-Niveau und bei acht davon auch zum 5\%-Niveau von Null verschieden. $^{48)}$ Bei der Betrachtung des Indexes läßt sich analoges feststellen, wobei in der vierten Perioden der US-Dollar sogar den einzigen Faktor darstellt, dem ein signifikanter Einfluß nachgewiesen werden kann. Ökonomisch begründet werden könnte die verstärkte Abhängigkeit der Kurse von Versicherungsaktien vom US-Dollar durch die gestiegene Integration der internationalen Finanzmärkte und der zunehmenden Rolle des US-Dollar als EURO-Außenwährung nach dem

45) So kam es im Zuge der Krisen in Asien, Lateinamerika und Russland im Sommer bzw. Herbst $1998 \mathrm{zu}$ einem erheblichen Einschnitt im Risikoverhalten internationaler Finanzinstitutionen, wie der Sell-Off von Finanzaktien und die plötzliche Flucht in Staatsanleihen zeigt; vgl. hierzu auch Tinker et. al. 1999.

46) Vgl. Mei/Saunders 1994, S. 20.

47) Vgl. im weiteren auch Entorf 2000, S. 520ff., der ähnliche empirische Befunde für den gesamten deutschen Aktienmarkt feststellen konnte.

48) Dabei sind es vor allem die großen und liquiden Werte, für die diese Signifikanz festgestellt werden kann. Bei den acht zum 5\%-Niveau signifikanten Gesellschaften handelt es sich um die Aachener und Münchener Leben, Allianz Holding, AXA Colonia Allgemeine und AXA Colonia Leben, DBV-Winterthur, Gerling-Konzern, Nürnberger und Volksfürsorge. 
Vertrag von Maastrich. ${ }^{49)}$ Dabei konzentrieren Analysten und Anleger ihre Gewinn- und Kurserwartungen in verstärktem Maße auf Bewegungen des Dollars und nicht mehr auf die Währungen der übrigen EU-Länder. Verstärkt werden könnte dieser für den gesamten deutschen Aktienmarkt wirkende Effekt durch die Akquisitionspolitik deutscher Versicherer im Ausland, insbesondere in den USA, was ein höheres Exposure zum US-Dollar bedeutet. ${ }^{50)}$

Der Januareffekt war jeweils nur für sehr wenige Aktien von Bedeutung, außerdem läßt sich auch kein eindeutiges Vorzeichen feststellen. Für den Index war in keiner der vier Betrachtungszeiträume signifikant von Null verschieden. Diese Ergebnisse ähneln den von Mei/Saunders, die ebenfalls keine Signifikanz der Januardummies feststellen konnten. ${ }^{51)}$ Ebenso ist die Sensitivität der Risikoprämien der hier betrachteten Versicherungsaktien bezüglich der nicht antizipierten Inflationsrate gering und nur selten statistisch signifikant. Auch dies korrespondiert mit vergleichbaren Studien für den amerikanischen Aktienmarkt. ${ }^{52)}$ Der Einfluß von Veränderungen der Industrieproduktion ist vergleichsweise oft signifikant. So konnten für den Index in den Unterperioden von 1975-1980 sowie 1987-1992 signifikante Koeffizienten beobachtet werden. Allerdings sind die betragsmäßigen Werte der Koeffizienten und damit das Ausmaß der Sensitivität der Risikoprämien von Versicherungsaktien gegenüber diesem Risikofaktor sehr gering.

\subsection{Prognose von Risikoprämien}

Ein wichtiges Einsatzgebiet von Multifaktorenmodellen in der Investmentpraxis ist die Prognose von Renditen von Aktien bzw. Aktienportfolios. Dabei werden die Prognosewerte dadurch ermittelt, indem die Faktorausprägungen - mit den aus der Regression bestimmten Faktorladungen gewichtet - addiert werden. Je näher die tatsächlichen Risikoprämien bei den prognostizierten Werten liegen, desto

49) Vgl. hierzu Entorf 2000, S. 531.

50) So erwarb etwa die Allianz AG im Jahre 1991,die Fireman's Fund Insurance Company, womit sie mit ihren US-Töchtern der Allianz of America und der North American Life and Casualty, über ein weites Filialnetz in Nordamerika und folglich einen substantiellen Umsatzanteil in US-Dollar verfügt; vgl. Liedtke 1996, S. 24.

51) Vgl. Mei/Saunders 1994, S. 20. Die Vergleichbarkeit der Ergebnisse für die einzelnen Aktien ist allerdings dadurch leicht eingeschränkt, daß die beiden Autoren ihr Modell nicht auf einzelnen Werten, sondern auf zwei Indizes basieren. Jedoch zeigt sich auch für den hier verwandten Index keine Signifikanz.

52) So etwa in Chen et al. 1986. 
höher die Prognosegüte des Modells. Insofern ist die Evaluation der Prognosegüte des im vorherigen Abschnitt spezifizierten Modells von Bedeutung.

Dabei sind zwei Arten von Abweichungsanalysen denkbar. Einerseits können die Faktorladungen aus der gleichen Periode benützt werden, was den „In-theSample-Test“" ergibt. Andererseits können die Faktorladungen aus der Regression der Vorperiode Eingang in die Berechnung der Modellrendite finden. Das letztgenannte Verfahren - auch „Out-of-the-Sample-Test“ genannt - ist das für praktische Anwendungen interessante, da hier nur Informationen verwendet werden, die zum Zeitpunkt der Prognoseerstellung auch tatsächlich verfügbar sind.

Zur Evaluation der Prognosegüte für die verschiedenen Teilperioden spezifizierten Modelle werden zwei quantitative Kennzahlen herangezogen: Zum einen wird berechnet mit welcher relativen Häufigkeit das Vorzeichen der prognostizierten mit den tatsächlichen Überrenditen einer Periode übereinstimmt. Zum anderen wird die mittlere quadratische Abweichung der in einem festgelegten Untersuchungszeitraum prognostizierten und realisierten Risikoprämien ermittelt. In der nachfolgenden Tabelle 8 sind diese beiden Kennzahlen differenziert nach Teilperioden für den Versicherungsindex angegeben.

Tabelle 8: Prognosegüte des Modells für den Index.

\begin{tabular}{lccc}
\hline \multicolumn{1}{c}{ Zeitraum } & $\mathbf{1 9 8 1 - 1 9 8 6}$ & $\mathbf{1 9 8 7 - 1 9 9 2}$ & $\mathbf{1 9 9 3 - 1 9 9 8}$ \\
\hline & Relative Häufigkeit korrektes Vorzeichen \\
\hline In-the-Sample & $66,67 \%$ & $66,67 \%$ & $62,50 \%$ \\
Out-of-the-Sample & $56,94 \%$ & $45,83 \%$ & $63,84 \%$ \\
\hline \multicolumn{4}{c}{ Mittlere quadratische } \\
\hline In-the-Sample & $0,337 \%$ & $0,379 \%$ & $0,119 \%$ \\
Out-of-the-Sample & $0,402 \%$ & $0,563 \%$ & $0,213 \%$ \\
\hline
\end{tabular}

Zunächst ist es nicht verwunderlich, dass die Prognosequalität der Out-of-theSample-Analyse nicht so gut wie bei dem „In-the-Sample-Test“ ausfällt. Trotzdem liegen die Modellvorhersagen nicht völlig von den tatsächlichen entfernt, wie das angesichts der Datenlage nicht überraschend gewesen wäre. ${ }^{53)}$ So weisen die prognostizierten Risikoprämien beim In-the-Sample-Test für alle Teilperioden in mehr als 50\% der Perioden das gleiche Vorzeichen wie der realisierte Indexwert auf. Mit Ausnahme der Teilperiode 1987-1992, die mit

53) Man bedenke in diesem Zusammenhang, daß die meisten der hier untersuchten Aktien relativ illiquide Werte sind. 
45,83\% eine nur geringe Trefferquote aufweist, gilt dies auch für die Out-of-theSample Abweichungsanalyse. Damit bietet sich der Einsatz des Modells im Rahmen von Market-Timing Strategien an. Die mittlere quadratische Abweichung der vom Modell vorgeschlagenen Werte mit den tatsächlichen Ausprägungen fällt in den beiden Teilperioden relativ hoch aus. Dies gilt erneut im Besonderen für die Out-of-the-Sample-Analyse bezogen auf den Zeitraum 1987-1992. Im Vergleich dazu fallen die Vorhersagen des Modells in der letzten Periode deutlich besser aus. Insgesamt ist zu konstatieren, dass die Risikoprämien des betrachteten Index aus Versicherungsaktien relativ gut durch das spezifizierte Faktormodel prognostizierbar sind.

\section{$5 \quad$ Zusammenfassung der Ergebnisse}

Im Zentrum dieser Arbeit stand die empirische Identifikation ökonomischer Einflussfaktoren auf die Risikoprämien deutscher Versicherungsaktien. Methodisch wurde ein Multifaktorenmodell mit makroökonomischen Variablen verwendet, welches gedanklich der APT folgt. Die Erklärungskraft des betrachteten Modells lieferte Ergebnisse, die im Rahmen vergleichbarer Studien für den US-amerikanischen Markt liegen resp. teilweise sogar etwas ausfielen. Dabei konnte insbesondere dem allgemeinen Zinsniveau ein negativer und dem Dollarkurs ein im Zeitablauf steigender positiver Einfluß auf die Risikoprämien der betrachteten Versicherungsaktiengesellschaften nachgewiesen werden. Ein für Investmentzwecke bedeutsames Resultat ist, dass die Risikoprämien der betrachteten Versicherungsaktien, trotz starker zeitlicher Schwankungen, relativ gut prognostizierbar sind. Erweiterungsmöglichkeiten der vorliegenden Studie bestehen neben dem Einsatz modifizierter Schätzverfahren vor allem in der Verwendung unternehmensspezifischer Erklärungsfaktoren. 


\section{Literaturverzeichnis}

Albrecht, P. (1991): Kapitalmarkttheoretische Fundierung der Versicherung ?, Zeitschrift für die gesamte Versicherungswissenschaft 80, S. 499-530.

Albrecht, P.; R. Maurer, J. Mayser (1996): Faktormodelle: Grundlagen und Einsatz im Management von Aktien-Portefeuilles, Zeitschrift für betriebswirtschaftliche Forschung 48, S. 3-29.

BARRA (1991): The German Equity Model, BARRA, Associates.

Beckers, S.; P. Cummins; C. Wood (1993): The Estimation of Multiple Factor Models and their Applications: The Swiss Equity Market, Finanzmarkt und Portfolio Management 7, S. 24-45.

Berry, M.A.; E. Burmeister; M.B. McElroy (1988): Sorting out Risks Using Known APT Factors, Financial Analysts Journal 44, S. 29-42.

Böcking, H.-J.; K. Nowak (1998): Der Beitrag der Discounted Cash Flow-Verfahren zur Lösung der Typisierungsproblematik bei Unternehmensbewertungen - Eine Warnung vor einer „,naiven“ Übertragung modelltheoretischer Erkenntnisse auf die Bewertungspraxis, Der Betrieb 51, S. 685-690.

Böcking, H.-J.; K. Nowak (1999): Marktorientierte Unternehmensbewertung: Darstellung und Würdigung der marktorientierten Vergleichsverfahren vor dem Hintergrund deutscher Kapitalmarktverhältnisse, Finanz-Betrieb 1, S. 169-176.

Burmeister, E.; R. Roll; S.A. Ross (1994): A Practitioner's Guide to Arbitrage Pricing Theory, Finanzmarkt und Portfolio-Management 8, S. 304-331.

Chamberlain, G. (1983): Funds, Factors and Diversification in Arbitrage Pricing Models, Econometrica 51, S. 1305-1323.

Chamberlain, G.; M. Rothschild (1983): Arbitrage and Mean Variance Analysis Pricing Models, Econometrica 51, S. 1281-1304.

Chen, N.-F.; R. Roll; S.A. Ross (1986): Economic Forces in the Stock Market, Journal of Business 59, S. 383-403.

Connor, G. (1995): The Three Types of Factor Models: A Comparison of their Explanatory Power, Financial Analysts Journal, March-June 1995, S. 42-46.

Cummins, J.D.; (1990): Asset Pricing Models and Insurance Ratemaking, ASTIN Bulletin 20, S. 125-166.

Cummins, J.D.; S.E. Harrington, Hrsg. (1987): Fair Rate of Return in Property-Liability Insurance, Boston.

Cummins, J.D.; S.E. Harrington (1988): The Relationship Between Risk and Return: Evidence for Property-Liability Insurance Stocks, Journal of Risk and Insurance 55, S. 15-31.

Derring, R. (1990): The Development of Property-Liability Insurance Pricing Models in United States 1969-1989, Proceedings on the $1^{\text {st }}$ AFIR International Colloquium, Vol. 4, S. 237-263.

Eberts, E.; R. Maurer (1999): Modelle zur Prognose der Inflationsrate, Mannheimer Manuskripte zu Risikotheorie, Portfolio Management und Versicherungswirtschaft Nr. 118, Universität Mannheim.

Entorf, H. (2000): Der deutsche Aktienmarkt, der Dollar und der Außenhandel, Zeitschrift für Betriebswirtschaft 70, S. 515-539.

Fama, E.F.; K.R. French (1992): The Cross-Sections of Expected Stock Returns, Journal of Finance 47, S. 427-465. 
Fama, E.F.; K.R. French (1993): Common Risk Factors in the Returns on Stocks and Bonds, Journal of Financial Economics, Vol. 33, S. 3-56.

Farny, D. (1983): Die Deutsche Versicherungswirtschaft, Karlsruhe.

Friend, I.; R. Westerfield; M. Granito (1978): New evidence on the Capital Asset Pricing Model, Journal of Finance 33, S. 903-916.

Frantzmann, H.-J. (1990): Zur Messung des Marktrisikos deutscher Aktien, Zeitschrift für betriebswirtschaftliche Forschung 42, S. 67-83.

Gesamtverband der Deutschen Versicherungswirtschaft - GDV - (1997): Statistisches Taschenbuch der Versicherungswirtschaft 1997, Karlsruhe.

Hachmeister, D. (1998): Der Discounted Cash-Flow als Maß der Unternehmenswertsteigerung, 2. Aufl., Frankfurt/M.

Harrington, S. E. (1983): The Relationship Between Risk and Return: Evidence for Live Insurance Stocks, Journal of Risk and Insurance 50, S. 587-610.

Haugen, R. A. (1997): Modern Investment Theory, 4. Aufl., Upper Saddle River, Prentice Hall, 1997.

Hill, R.D.; F. Modigliani (1987): The Massachusetts Model of Profit Regulation in Nonlife Insurance: Appraisal and Extension in: J.D. Cummins; S.E. Harrington (Hrsg.), Fair Rate of Return in Property-Liability Insurance, Nowell, MA: Luwer Academic Publishers 1987.

Huberman, G. (1982): A Simple Approach to Arbitrage Pricing Theory, Journal of Economic Theory 28, S. 183-191.

IDW ES 1 (1999): Entwurf IDW Standard: Grundsätze zur Durchführung von Unternehmensbewertungen, Die Wirtschaftsprüfung 52, S. 200-216.

Ingersoll, J.E. (1987): Theory of Financial Decision Making, Savage, ML, Rowman \& Littlefield, 1987.

Kempf, A. (1998): Was messen Liquiditätsmaße? Die Betriebswirtschaft 58, S. 299-311.

Kleeberg, J.M. (1992): Der Einsatz von fundamentalen Betas im modernen Portfoliomanagement, Die Bank, S. 474-478.

Kraus, A.; S. Ross (1982): The Determinants of Fair Profit Rates for the PropertyLiability Insurance Firm, Journal of Finance 37, S. 1015-1028.

Kvålseth, T.O. (1985): "Cautionary Note About R2", The American Statistican. 39, S. 279-285.

Lee, A.C.; D. Cummins (1998): Alternative Models for Estimating the Cost of Equity Capital for Property/Casualty Insurers, Review of Quantitative Finance and Accounting 10, S. 235-267.

Liedtke, R. (1996): Wem gehört die Republik? Die Konzerne und ihre Verflechtungen: Namen, Zahlen, Fakten, Frankfurt/M.

Lintner, J. (1965): The Valuation of Risky Assets and the Selection of Risky Investments in Stock Portfolios and Capital Budgets, Review of Economics and Statistics 47, S. 13-37.

Mack, T. (1997): Schadenversicherungsmathematik, Karlsruhe 1997.

Mayser, J. (1996): Aktive Aktien-Portefeuillesteuerung auf der Grundlage stochastischer Ansätze, Aachen 1996.

Mei, J; A. Saunders (1994): The time-variation of risk premiums on insurer stocks, Journal of Risk and Insurance 61, S. 12-32.

Möller, H.P. (1985): Bilanzkennzahlen und Ertragsrisiken des Kapitalmarktes, Stuttgart. 
Montgomery, D.C.; E.A. Peck, (1982): Introduction to Linear Regression Analysis, New York.

Mossin, J. (1966): Equilibrium in a Capital Asset Market, Econometrica 34, S. 768-783.

Nielsen, L. (1992): Quantifizierung von Investitionsrisiken auf dem Deutschen Aktienmarkt, Die Bank, S. 228-230.

Oesterhelweg, O.; D. Schiereck (1993): Meßkonzepte für die Liquidität von Finanzmärkten, Die Bank, S. 390-397.

Rao, C. R. (1996): Principal Component and Factor Analysis in: Maddala, G. S.; C. R. Rao, (Hrsg.): Handbook of Statistics 14, S. 489-505, Amsterdam.

Roll, Richard (1977): A Critique of the Asset Pricing Theory“s Tests, Part I: On Past and Potential Testability of the Theory, Journal of Financial Economics 4, S. 129-176.

Roll, Richard; Stephen A. Ross (1980): An empirical investigation of the arbitrage pricing theory, Journal of Finance 35, S. 1073-1103.

Roll, R.; S. A. Ross (1995): The Arbitrage Pricing Theory Approach to Strategic Portfolio Planning, Financial Analysts Journal, January/February 1995, S. 122-131.

Rosenberg, B. (1974): Extra-Market Components of Covariance in Security Returns, Journal of Financial and Quantitative Analysis 9, S. 263-274.

Rosenberg, B.; V. Marathe (1976): Common factors in Security Returns: Micro economic determinants und macro correlates, Proceedings of the seminar on the analysis of security prices, University of Chicago, S. 61-116.

Ross, S. A. (1976): The arbitrage theory of capital asset pricing, Journal of Economic Theory 13, S. 341-360.

Rozeff, M.S.; W.R. Kinney (1976): Capital Market Seasonality: The Case of Stock Returns, Journal of Financial Economics 3, S. 379-402.

Rudd, A.; H.K. Clasing (1988): Modern Portfolio Theory: The Principles of Investment Management, 2. Aufl., Orinda.

Arbeitskreis „Finanzierung“ der Schmalenbach-Gesellschaft Deutsche Gesellschaft für Betriebswirtschaft e.V. (1996): Wertorientierte Unternehmenssteuerung mit differenzierten Kapitalkosten, Zeitschrift für Betriebswirtschaftliche Forschung 48, S. 543-578.

Schwetzler, B. (2000): Unternehmensbewertung unter Unsicherheit Sicherheitsäquivalent- oder Risikozuschlagsmethode?, Zeitschrift für betriebswirtschaftliche Forschung 52, S. 469-486.

Shanken, J. (1982): The arbitrage theory: Is it testable ?, Journal of Finance 37, S. 11291140.

Stephan, T.G.; R. Maurer (2000): Multi-Faktor-Modelle zur Steuerung von Aktienportfolios, Handbuch...

Steffen, K.-J. (1967): Preisbestimmende Faktoren und Preisbildung für Versicherungsaktien an der Börse, Band 3, Berlin.

Steiner, M.; C. Bauer (1992): Die fundamentale Analyse und Prognose des Marktrisikos deutscher Aktien, Zeitschrift für betriebswirtschaftliche Forschung, 44, S. 347-368.

Tinker, M.; M. O’Sullivan; G. Secker (1999): Stocks and interest rates - volatility contagion, in: Warburg Dillon Read (Hrsg.): Special Equity Briefing, 26th October 1999, S. 1-20.

Wei, K.C.J. (1988): An Asset Pricing Theory Unifying the CAPM and APT, Journal of Finance 43, S. 881-892. 